\title{
Mythos
}

Rivista di Storia delle Religioni

13 | 2019

Varia

\section{L'officina mitologica di Furio Jesi. Sulle prefazioni non pubblicate a Materiali mitologici}

Furio Jesi's mythological workshop. The unpublished forewords of Materiali mitologici

\section{Enrico Manera}

\section{OpenEdition}

\section{Journals}

\section{Edizione digitale}

URL: http://journals.openedition.org/mythos/996

ISSN: 2037-7746

\section{Editore}

Salvatore Sciascia Editore

Notizia bibliografica digitale

Enrico Manera, «L'officina mitologica di Furio Jesi. Sulle prefazioni non pubblicate a Materiali mitologici », Mythos [Online], 13 | 2019, online dal 01 décembre 2019, consultato il 26 décembre 2019. URL http://journals.openedition.org/mythos/996

Questo documento è stato generato automaticamente il 26 dicembre 2019

Mythos 


\title{
L'officina mitologica di Furio Jesi. Sulle prefazioni non pubblicate a Materiali mitologici
}

Furio Jesi's mythological workshop. The unpublished forewords of Materiali mitologici

\author{
Enrico Manera
}

\section{NOTE DELL'AUTORE}

Questo scritto è dedicato alla memoria di Marta Rossi Jesi (1936-2018). Rinnovo il ringraziamento alla famiglia Jesi per il sostegno e l'attenzione dedicati alle mie ricerche. Grazie a Pierluigi Lanfranchi per la lettura del testo e lo scambio di idee.

La mia fiducia viene dalla convinzione che tutti gli esseri umani si somigliano, che altri portano ferite come le mie e che quindi capiranno. Tutta la vera letteratura nasce da questa certezza fiduciosa e infantile che tutti gli individui si somiglino. Quando uno scrittore si chiude per anni in una stanza, evoca con il suo gesto l'esistenza di un'umanità unica, un mondo privo di centro.

O. Pamuk, La valigia di mio padre (ed. or. 2006), trad. it., Torino 2007, 20.

\section{Evitare il gorgo: la meccanica mitologica}

1 Nel suo lavoro interdisciplinare di ricerca e scrittura Furio Jesi (1941-1980), noto per i suoi lavori come storico delle idee, critico letterario e germanista, si è costantemente interrogato sugli aspetti teoretici, linguistici e simbolici che riguardano il mito, i miti e la mitopoiesi nella storia della cultura ed è approdato alla proposta di un modello gnoseologico originale, rielaborato in più fasi e rigorosamente provvisorio, denominato «macchina mitologica». Questa è descritta come una «immagine [...] per definire la forma di un congegno che produce forme di epifanie di miti e che nel suo interno, di là dalle sue pareti non penetrabili, potrebbe contenere i miti stessi - il mito -, ma 
potrebbe anche essere vuoto»"; qualcosa "che produce mitologie e induce a credere, pressante, che essa stessa celi il mito entro le proprie pareti non penetrabili» ${ }^{2}$. Nel volume Mito (1973) Jesi ha sostenuto che, per evitare che diventi «un centro fascinatorio» tale da «esigere prese di posizione», la macchina mitologica deve essere accolta come «un puro modello funzionale e provvisorio»" prioritario è infatti «indagare il funzionamento dei meccanismi della macchina mitologica» e mettere «fra parentesi» ${ }^{4}$ le questioni relative all'essere del mito.

2 Jesi definisce «la "scienza del mito"» come «scienza di ciò che non c'è $»^{5}$. La parola "mito" indica un oggetto culturale che viene spesso inteso come sostanza, anche se che non può essere considerato come tale: molte teorie infatti lo concepiscono implicitamente o esplicitamente come la (presunta) epifania di forze extra-umane che si presentano alla coscienza umana. In tal modo la mitologia si configura nella storia della cultura come sapere metafisico che promette l'accesso privilegiato a verità celate e il mito, le cui caratteristiche sarebbero genuinità, forza ed eterna presenza, assume l'aspetto di una realtà infinita e invisibile. Così avviene ad esempio nel pensiero di Walter $\mathrm{F}$. Otto o Mircea Eliade, nelle quali esso appare come epifania o teofania che, manifestandosi, «afferra la vita e la plasma» ${ }^{6}$.

3 Contro ogni impropria ipostatizzazione la proposta teorica jesiana consiste in un avvicinamento per via negativa al mito - da intendersi come oggetto elusivo, distante dallo studioso e sepolto nel passato - alla ricerca delle tracce della sua assenza. Quella del mito è dunque scienza del non-esserci, cioè di qualcosa che esiste nell'immaginario $\mathrm{e}$ assume importanti significati di ordine esistenziale e comunitario: dotato di valore metafisico e religioso, il mito infatti tende a certificare come realtà i propri contenuti attraverso credenze e azioni di chi in esso si riconosce. Quale che sia il loro contenuto, $\mathrm{i}$ miti circolano, si mostrano come verità di ordine superiore, modellano universi mentali e generano conseguenze ${ }^{7}$.

4 "Il mito" deve essere considerato come non-sostanza a meno che non si voglia fare della sua scienza «un cavallo di Troia per introdurre nel recinto delle analisi dei materiali culturali una sorta di ierologia» ${ }^{8}$. Nella prospettiva di Jesi - intellettuale impegnato nella "nuova sinistra" italiana degli anni Settanta che tra i suoi riferimenti annovera Kerényi, Mann, Cassirer, Benjamin - compito del mitologo è sottrarsi in modo particolare al gorgo in cui è precipitata la cultura europea nel Novecento a causa della manipolazione ideologica operata sul mito dalla "tecnicizzazione fascista", l'uso politico del mito che appare come tratto tipico della cultura di destra ${ }^{9}$. La riflessione dovrà prima di tutto bonificare i propri presupposti teorici per non «confondersi con l'elaborazione dottrinale della mistica del potere» ${ }^{10}$.

5 Il saggio La festa e la macchina mitologica, pubblicato nel 1973, nel 1977 e poi nella raccolta Materiali mitologici. Mito e antropologia nella cultura mitteleuropea $(1979)^{11}$, è da questo punto di vista uno dei testi cruciali per gli aspetti teorici dell'opera jesiana e può essere considerato come uno degli assi portanti dell'opera matura dell'autore. La festa è presa in considerazione come "fatto sociale totale", sulla scorta di una lunga tradizione culturale e in ragione della sua stretta parentela con la dimensione mitica. Jesi sottolinea in queste pagine come sia venuta meno nella modernità la funzione di fondazione sacralizzante e coesiva per la comunità che il racconto mitico, coestensivo al momento festivo, ha avuto nel mondo antico; fin dal XVIII secolo una prima elaborazione di indagine etnologica spinge gli studiosi europei a studiare forme di umanità percepite come un altro da sé: studiare i "primitivi viventi” postulando la loro 
somiglianza con gli antichi implica l'impossibilità per l'osservatore, esterno e lontano, di rapportarsi con l'esperienza vissuta da una collettività altra e in stato festivo. Gli etnografi - scrive Jesi - «videro le feste dei diversi, non videro ciò che i diversi vedevano. Li videro vedere, non videro l'oggetto della visione, o almeno non lo videro con gli occhi dei veggenti ma solo con gli occhi dei voyeurs» ${ }^{12}$.

Questa condizione è considerata paradigmatica dello studioso moderno nei confronti del mito e dei fenomeni religiosi. Si tratta di un giudizio nel quale il pensiero di Kerényi ${ }^{13}$, che Jesi riconosce come maestro pur allontanandosene, appare radicalizzato: ogni rapporto "vivo", in flagranti, con il mito si rivela infatti impossibile e non può che essere oggetto di una ricostruzione fatta dallo studioso a partire dalle sue griglie interpretative, parziali e soggettive ${ }^{14}$; inoltre, per effetto di un processo di desacralizzazione, l'arte e letteratura si configurano come attività mitopoietiche e come modalità di produzione di nuovi materiali mitologici all'interno di un lavoro di ricezione e rielaborazione nell'ambito di processi culturali di cui l'oggetto "mito" è protagonista $^{15}$.

7 La perduta "festa di ieri" è dunque intesa come modello del vissuto religioso: se questa aveva al suo centro l'«istante privilegiato dinanzi al disvelarsi dell'eidolon», nel momento in cui "gli dèi si sono allontanati "nelle profondità del loro nulla" [...] l'eidolon ha cessato di esistere e con esso la visione [...], l'illusione ottica dell'orizzonte dello spazio tra uomini e dèi è cessato» ${ }^{16}$. Nella modernità quindi la festa e il mito si presentano nei termini di un rapporto con ciò che non si può dare, sulla base di una razionalità segnata dal tempo storico: per questo nel pensiero di Jesi lo studio del passato è conoscenza problematica di ciò che è assente, distante ed opaco, tale da escludere quella "commozione" o partecipazione empatica che è stato il presupposto dalle scienze religiose otto-novecentesche, se non per un distorto effetto nostalgico e intrinsecamente conservatore.

Dove molti mitologi cercano la sostanza del mito, la critica jesiana vede solo «una macchina che produce mitologie e che genera la tenace illusione di nascondere il mito entro le proprie imperscrutabili pareti $\aleph^{17}$ : con l'espressione "macchina mitologica" l'esito più rilevante di una epistemologia della scienza del mito - si intende non solo un originale modello gnoseologico ma l'intero rapporto della cultura moderna con l'immagine di un (inesistente) passato perduto e migliore, spiritualmente vivo e comunitariamente pieno di significati. Nelle formulazioni dell'autore tale processo gnoseologico, culturale e sociale appare simile a un congegno che produce «fatti mitologici» e la cui prima funzione è «indicare qualcosa che non può essere visto» ${ }^{18} \mathrm{e}$ che si vuole primitivo, originale, vivo ${ }^{19}$ : la macchina mitologica

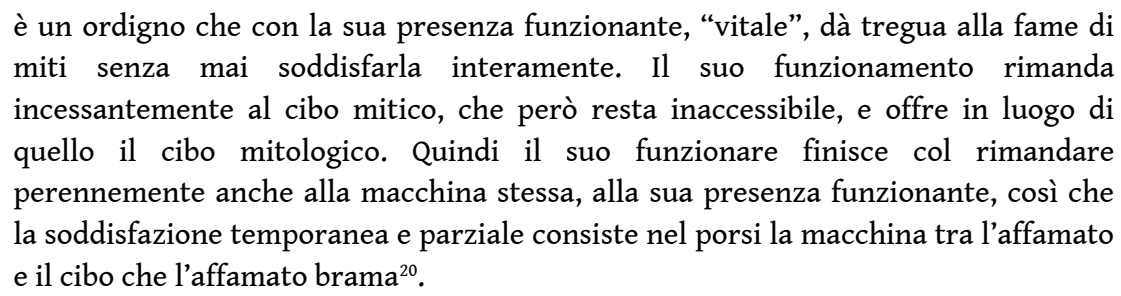

9 La metafora della macchina descrive un funzionamento di tipo "automatico", tale da fornire momentaneo sollievo alla «fame di mito» in quanto supposto "ens quatenus ens»; allo stesso tempo pone in dubbio "questa determinazione ontologica del mito» nel momento in cui la macchina «produce mitologie» cioè «entes in quanto prodotte dalla 
macchina ${ }^{21}$ stessa. La macchina mitologica svolge una funzione di fondazione e di produzione di senso che risulta prima di tutto relativa alla propria genesi:

la macchina mitologica è autofondante: pone la sua origine nel fuori di sé che è il suo interno più remoto, il suo cuore di pre-essere, nell'istante in cui si pone in atto. Questo presupponimento di origine (il rimando al mito) è totalizzante: coinvolge tutti gli istanti e gli ambiti spaziali di funzionamento della macchina, poiché il fuori di sé in cui la macchina pone la sua origine è il suo centro. Ogni fatto mitologico è quindi esso stesso presupponimento della propria origine, che è altresì l'origine della macchina ${ }^{22}$.

È opinione di chi scrive che in Jesi il discorso metafisico vada riportato sul piano antropologico: la mitopoiesi è un processo dinamico nel quale l'idea secondo cui "il mito afferra l'uomo" vada rovesciata in favore di quella secondo cui le figure mitiche che si presentano alla coscienza umana sono indipendenti da presunti correlati esterni; le immagini mitologiche sono etimologicamente finzioni e invenzioni, storiche e storicamente trasmesse, che gli esseri umani producono per pensare se stessi nel mondo. Il concetto ipostatizzato di "mito" va visto come il risultato di una costruzione culturale che occulta il suo operare e lo naturalizza; la sua sostituzione con la nozione di "macchina mitologica" risulta in grado di mostrare e storicizzare il tessuto ideologico che produce i "materiali mitologici": in altri termini, i miti sono storie che non possono essere comprese se non in relazione agli uomini che li raccontano ${ }^{23}$.

\section{Nell'officina del mitologo: materiali d'archivio}

La "macchina mitologica" è il nodo principale di un'opera sui problemi relativi alla scienza del mito a cui Jesi ha lavorato a partire dai primi anni Settanta e di cui diverse parti sono confluite nella redazione finale della raccolta di saggi Materiali mitologici. I documenti conservati nell'archivio privato testimoniano possibili varianti e trasformazioni nel corso del tempo, lasciando intravvedere la progettazione di un'opera complessa e di non facile scrittura per la vastità di argomenti previsti e per la scelta dei saggi dalla cui interazione sarebbe dovuto risultare il quadro finale. Come scrive Andrea Cavalletti, a cui si deve un attento lavoro di scavo dell'archivio,

deve essere riconosciuto in questo lavorìo continuo di aggregazioni, poi di successive scomposizioni e ricostruzioni, la maniera più caratteristica dell'officina jesiana, propria di un pensiero che si trasmette sempre per via indiretta, e le cui tracce sono da individuare, secondo un'indicazione preziosa "non nel 'contenuto' dei miei scritti, ma nella loro architettura, nei loro criteri compositivi, in ciò che li fa essere 'composizioni' (nel senso musicale della parola)" 24 .

Lo stile jesiano si caratterizza infatti per una «tecnica di "composizione" critica di dati e dottrine, fatti reagire tra di loro, il cui modello metodologico si trova nella formula del conoscere per citazioni [...] di W. Benjamin $»^{25}$. Inoltre la comparazione tra mitologia e facoltà musicale - un tema caro tanto a Kerényi quanto a Lévi-Strauss - che si fonda sulla concezione della mitologia in quanto "arte" e in quanto "materiale", sembra venire estesa allo studio della mitologia come sua continuazione ${ }^{26}$. Per Jesi la scienza che nasce nel XVIII secolo con la sua elaborazione del mito sarebbe cioè un prolungamento della mitologia stessa e processo ulteriore di nuova mitizzazione. Sulla base di un'analisi complessiva del suo lavoro si può osservare come il mitologo torinese abbia inteso il rapporto tra testo e commento come seguito dell'opera, collocandosi così nell'alveo che connette cultura romantica, ermeneutica e decostruzione. In questo 
senso, il moltiplicarsi dei piani di lettura e i continui rimandi suggeriti dalla tessitura delle citazioni sono aspetti caratterizzanti la scrittura dello Jesi maturo.

I tredici saggi che compongono la raccolta Materiali mitologici rivelano la loro parentela quando se ne colga l'aspetto di ricognizione su diversi momenti della ricezione della mitologia nella storia della cultura: un sondaggio compiuto principalmente nel campo della letteratura di lingua tedesca del primo Novecento e nel suo riferimento all'antico. Il libro nella sua versione finale è stato pubblicato senza introduzioni, come se l'autore volesse rinunciare a ogni spiegazione preliminare ${ }^{27}$; sembra significativo inoltre che Jesi abbia evitato un titolo focalizzato sulla macchina - che avrebbe promesso una teoria ben definita, onnicomprensiva ed esplicativa - in favore del definitivo Materiali mitologici.

14 L'edizione del volume del 2001 presenta in Appendice una serie molto significativa di testi provenienti dall'archivio che sono l'oggetto dell'analisi nelle pagine seguenti: si tratta di una introduzione e sei differenti prefazioni redatte su fogli dattiloscritti contenuti in una cartella intitolata La macchina mitologica, le quali, a dispetto della tendenziale compiutezza, sono state scartate ${ }^{28}$. Quel materiale, databile non oltre il 1976, si configura come uno sguardo sul backstage dell'officina del mitologo e la sua analisi permette di cogliere importanti elementi sulla genesi teorica del metodo jesiano. I testi mostrano infatti alcune riserve espresse nei confronti dell'idea di consegnarsi alla definitività della concettualizzazione: nel leggere in sequenza queste ipotetiche e mancate soglie introduttive si ha l'impressione di trovarsi di fronte alla mobilità dei pensieri dell'autore e al disagio per il rischio di non riuscire a comunicare pienamente la totalità dei piani di cui tenere conto: le diverse varianti e i cambi di stile delle prefazioni - dalla prima alla terza persona, il privilegiare un aspetto su un altro suggeriscono l'indecisione sul discorso che avrebbe dovuto fissare, nel modo meno infedele possibile, quella stagione di riflessione.

Nel testo intitolato Introduzion $e^{29}$ è predominante un taglio gnoseologico: riferendosi al saggio La festa e la macchina mitologica Jesi riconosce come la sua proposta appaia un «modello "spettrale" " ${ }^{30}$ il quale, più che descrivere un vero e proprio funzionamento, circoscrive «il complesso delle necessità empiriche che ne sollecitano la formulazione»: ovvero la motivazione di natura anti-ideologica che ne fa uno strumento utile ad aggirare i limiti conoscitivi posti dalla mitologia. Limite indica qui ciò che delimita $\mathrm{e}$ divide due ambiti:

il modello macchina mitologica, nel quadro delle mie ricerche, resta efficiente, cioè soddisfa nel modo migliore i bisogni gnoseologici che ho avvertito studiando la mitologia, riempie adeguatamente la loro forma in cavo, solo nella misura in cui non diviene una forma a tutto tondo. [...] Esso resta efficiente, adoperabile solo finché la sua colata nell'impronta di quei bisogni è materia così esigua da formare una sottilissima pellicola aderente all'impronta, e tale da riprodurne fedelmente la cavità, anziché essere un flusso abbondante che riempie ogni cavo, lo colma, statuario $^{31}$.

16 Il problema di metodo che conduce a formulare la macchina è la necessità di una «pellicola» che separi il bisogno della conoscenza e la materia da conoscere. «La pellicola non è eliminabile» e serve a evitare il «cortocircuito» che si creerebbe dal «contatto diretto» tra la mitologia e la nostra «facoltà di creazione e percezione della mitologia»:

Quello che chiamiamo il mito, senza potere in termini di scienza avanzare alcuna ipotesi sulla sua natura e, anzi sulla sua effettiva esistenza, si incontrerebbe con i 
prodotti di quella che chiamiamo macchina mitologica: con i materiali mitologici, la mitologia. Per quanto abbastanza imprevedibili, le modalità e le conseguenze di questo corto circuito sembrano estremamente pericolose per la ragione ${ }^{32}$. riconosciuta alla mitologia una funzione di «amplificazione della personalità» ${ }^{38}$ : in questo senso, la mitologia appare capace di farsi «breccia attraverso la quale la personalità ricada nella pura esperienza irrazionale dell'essere e vi si annulli»». Ancora una volta si può leggere il rifiuto di una prospettiva metafisica e co-estensiva delle culture reazionarie, estranea alla coscienza critica e ragionante. Questa è l'istanza fondamentale anche della Prefazione (1) ${ }^{40}$ nella quale si legge che la «volontà di fondo» che accomuna i saggi che compongono il libro consiste nell'

affrontare lo studio dei materiali mitologici in modo che appaia senza riserve brutale e deformante [...] ogni operazione gnoseologica che vi scorga l'ipostasi di un assoluto metafisico effettivamente esistente ed operante ${ }^{41}$.

21 Jesi scrive qui che produzione e ricorso al mito, le «tecnicizzazioni fasciste dei materiali mitologici», costituiscono «un comportamento elementare» dell'umano che si qualifica come reazionario perché punta «a soddisfare il bisogno sfruttando gli altri uomini e, poi di conseguenza, a perpetuare le azioni di sfruttamento» ${ }^{42}$. Così ogni mitologia del potere rientra nell'ordine dell'«apparato ideologico e persuasivo»: a essa si contrappone la mitologia umanizzata proposta da Thomas Mann, sorta dal bisogno di «trasfunzionare» il mito e «toglierlo di mano dal fascismo» ${ }^{43} \mathrm{e}$ in grado di restituire l'unica "autenticità" possibile al materiale mitologico, ovvero quella derivante dal suo significato storico e umano.

Non è possibile qui esporre adeguatamente l'importanza che Thomas Mann ricopre nell'opera del critico e germanista torinese: basterà ricordare che a lui sono dedicati una monografia omonima e tre saggi dei Materiali mitologici ${ }^{44}$. La dimensione umanistica e antimetafisica della mitopoiesi appare decisiva: la figura del romanziere coincide 
infatti con la macchina mitologica della scrittura capace di produrre materiali di estrema durevolezza, indifferenza al tempo e alto tasso di significatività e tale da generare l'effetto di "eterna presenza" che costituisce la qualità principale della "miticità". Il mondo immaginario, che pare soprannaturale allo sguardo del tout se tient o della "sincronicità", è di fatto il più umano che ci sia: la letteratura è un universo denso di affettività in cui uomini e donne condividono esperienze simili e imparano a conoscere se stessi e gli altri attraverso i libri e le fitte reti inter-testuali. Lo spazio letterario finisce così per essere assimilato a un iper-luogo ideale, un altrove in cui è piacevole sostare e che - mitologicamente - potrà essere assimilato al mondo archetipico o all'eterno ritorno dell'uguale ${ }^{45}$. In ragione di questa «sorta di solidarietà universale $»^{46}$ tra i testi il critico letterario ha nella sua memoria una personale sintesi della letteratura universale.

In questo senso, Jesi fornisce in uno dei saggi di Materiali mitologici una delle più folgoranti descrizioni della modalità operativa della macchina mitologica:

Il libro è il mito incarnato, e se non tutti i libri si prestano a confermare questa sorta di aforisma, ciò dipende dalla violenza che viene operata sul libro, tanto da trasformarlo in oggetto di consumo. Il libro è il mito incarnato, nella misura in cui è, come un materiale mitologico, il prodotto di un congegno simbolico dalle pareti impenetrabili: di una macchina che produce mitologia e dice di contenere la verità segreta, non verificabile, il motore immobile, il mito in sé e per sé, di là dalle sue epifanie ${ }^{47}$.

\section{Storicizzazione integrale}

La Prefazione (2) ${ }^{48}$ dichiara la necessità di un metodo rigorosamente storico-culturale per lo studio del mito, del simbolo e della mitologia: l'unica conoscenza praticabile in questi ambiti è quella consapevole «della lontananza, dell'alterità e della frattura non guaribile» ${ }^{49}$. Jesi afferma inoltre che il «mito» appartiene all'ambito «dei nomi di quelle supposte realtà o entità, o quid riposanti in se stessi, cui appartiene ad esempio il nome "Dio"»: termini come "mitologia" o "materiali mitologici" sono più adatti per chi, come lui, non vive «un'esperienza religiosa tale da implicare un "Dio"»"

La coscienza della distanza ${ }^{51}$ dell'oggetto di studio implica anche consapevolezza dell'ampio margine di «indeterminazione», di arbitrarietà e contingenza che caratterizza i risultati delle scienze umane. La fenomenologia degli oggetti culturali di cui viene fatta professione è caratterizzata tanto dall'impossibilità della coincidenza «con l'esperienza di chi creò quei materiali, o [...] li sentì affiorare spontaneamente da

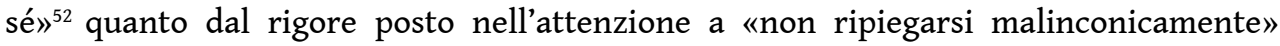
nell'apologia del passato che caratterizza il tratto luttuoso della filologia: si tratta di applicarsi

con la maggior finezza possibile, non al corpo di Lazzaro redivivo, ma al cadavere di Lazzaro. È se non altro materia da conoscere, una sostanza presente, con tutte le sue tossine cadaveriche, nell'ambiente che ci circonda. Sarebbe irresponsabile dichiarare che non c'è, e pericolosamente illusorio dichiarare che è sostanza viva, non in dissoluzione $\mathrm{e}^{53}$.

Il correlato del rifiuto di ogni illusoria presenza o partecipazione con il passato è un cauto avvicinamento di tipo storico-storiografico e critico-ideologico, come attesta la metafora del mito come corpo in decomposizione dagli effetti "tossici" perché connessi 
alla "religione della morte" e alla tecnicizzazione reazionaria e fascista che segna la cultura europea nel XIX e nel XX secolo.

Anche la successiva Prefazione (3) ${ }^{54}$ ruota attorno alla storicità: qui è sottolineata l'irriducibile particolarità di ogni momento della storia della mitologia, visto come episodio di funzionamento particolare della macchina mitologica. Da questo deriva il carattere "provvisorio e pratico» del modello, che vale «per la particolarità e la frammentarietà delle situazioni esaminate» ${ }^{55}$.

Egli [l'autore] non presume affatto che le sue conclusioni, quelle a suo parere valide per le situazioni che ha esaminato, possano essere legittimamente estese molto al di là di tali situazioni specifiche. Varranno, sì, per una certa area di situazioni analoghe, magari di cospicua ampiezza, ma sarebbe temerario supporre una loro qualsiasi applicabilità e validità universale ${ }^{56}$.

Il passato appartiene alla dimensione storica, allo stesso modo in cui alla storicità è soggetto anche il presente, cioè il tempo da cui ci si rivolge al passato; è da notare come Jesi assegni validità limitata e circoscritta anche alla macchina mitologica, il modello che gli consente di svolgere la sua analisi: «il modello ha presumibilmente un momento di applicabilità nella storia della ricerca scientifica. Il che non implica però la fiducia dell'autore [...] in un continuum storico, neppure o tanto meno al livello di una storia del pensiero scientifico» ${ }^{57}$. Il paradossale "ritorno alla vita" di un materiale mitologico, nella forma raffreddata dello studio, è condizionato dalla sua ricezione: allo stesso modo la macchina mitologica assume significato e produce senso per il momento storico della sua formulazione come modalità cognitiva necessaria in relazione ai bisogni del tempo a cui appartiene.

Il testo insiste sul rifiuto di proporre una teoria generale della cultura:

Se per comodità, per la facilità [...] con cui il linguaggio tende a essere definitorio $\mathrm{e}$ assoluto, $\mathrm{o}$ anche solo per l'opportunità di non rendere il discorso continuamente irto di dichiarazioni dei suoi limiti, l'autore si è lasciato sfuggire parole o tonalità da scopritore di leggi eterne e universali, egli ne fa qui ammenda ${ }^{58}$.

Così la successiva Prefazione (4) ${ }^{59}$ :

la "scienza della mitologia" è per eccellenza scienza del presente. Il suo oggetto è costituito dai materiali mitologici di cui disponiamo oggi, nell'istante in cui li esaminiamo; i suoi strumenti sono i nostri presenti modelli gnoseologici. I miti del passato e del futuro, i materiali mitologici del passato e del futuro, restano necessariamente estranei, bui, ignoti, per questa scienza ${ }^{60}$.

Qualora questo non fosse, la scienza cesserebbe di essere tale e diventerebbe «mistica, fede, profezia» o qualsivoglia altra «esperienza totalizzante» ${ }^{61}$. È evidente in questi passi il disagio che Jesi avverte di fronte alle prospettive filosofiche e storico-religiose che celano l'ambizione di essere ontologie metastoriche e filosofie dal portato esistenziale e politico ${ }^{62}$. Per Jesi "ciò che conta, nell'istante in cui il "materiale mitologico" cade sotto l'indagine, è l'attualità del modo di conoscerlo» ${ }^{63}$.

Mentre la nozione di "mito" ha fornito alla classica scienza che se ne è occupata elementi per la formulazione di presupposti indimostrabili connessi all'idea di universalità, lo studio della macchina mitologica opera mediante l'esposizione dei suoi frammenti e diviene nei fatti analisi decostruttiva condotta nella ricezione del presente, solo in questo senso in grado di illuminare costellazioni di senso. Del "mito" è possibile solo circoscrivere la sopravvivenza alterata, il riflesso scritto, manipolato, ideologizzato, interpretato, risemantizzato e inseparabile dal modello teorico in cui lo si inscrive. 


$$
\begin{aligned}
& \text { senso storico, teoretico, antropologico ed estetico quando diviene indagine sui } \\
& \text { materiali mitologici e si fa scienza del presente. Se "ciò che conta [...] è l'attualità" del } \\
& \text { modo di conoscere, l'opera di Jesi si configura come la personale declinazione di una } \\
& \text { teoria ermeneutica e della ricezione: } \\
& \text { Il mito è letto da Jesi come un rapporto dialogico tra testo e lettore/interprete. } \\
& \text { Rapporto che si situa in un "vuoto". In quel vuoto stanno le "epifanie del mito" e } \\
& \text { non il mito. Dunque è con le epifanie del mito che il soggetto intrattiene rapporti, in } \\
& \text { una condizione di costante dislessia perché lette come "miti genuini", epifanie che } \\
& \text { funzionano come una macchina desiderante: quanto più si innalza la "domanda" di } \\
& \text { mito, tanto più saranno "offerte" epifanie del mito, e quindi mitologie }{ }^{64} \text {. }
\end{aligned}
$$

Nella Prefazione (5) ${ }^{65}$ oltre alla nuova chiarificazione dei concetti di mito («presunta sostanza o ipostasi metafisica cara alle manipolazioni della cosiddetta destra tradizionale») e mitologia, («i materiali mitologici, l'oggetto e i risultati di molteplici esperienze, creazioni e tradizioni culturali») si legge l'intenzione di affrontare la questione presentando «indagini [...] più sperimentali che teoriche» tali da arricchire la «prassi» della «scienza della mitologia» ${ }^{66}$. Un approccio micrologico e per frammenti appare più produttivo - portatore cioè di nuova conoscenza - per le scienze umane, in alternativa a «teoresi», «osservazione» e «apprezzamento fenomenologico», definiti come differenti «paradisi degli archetipi» ${ }^{67}$ ormai perduti e irraggiungibili.

Jesi formula dunque per via negativa i presupposti del suo metodo, indicando le prospettive teoriche in cui non si riconosce: il suo sguardo sorge da una consapevolezza «calata ben dentro alla storia politica della nostra cultura, serve a mantenere distanze sia verso lo heideggeriano "crepuscolo dell'Occidente", sia verso gli ancora più ottimistici programmi di sistemazioni strutturalistica o semiotica delle forme di cultura e di vita» ${ }^{68}$. Nel dibattito coevo la sua strada si colloca tra i rischi opposti in cui incorre la scienza della mitologia. Da un lato si tratta di non affidare al mito significati ultimi, compito che sarebbe funzionale alla reazione politica alimentando forme gnostiche in cui la segretezza diviene di per sé valore. La filosofia di Heidegger è qui l'esempio di una mitizzazione volta a «riconsacrare il cosmo» ${ }^{69}$ : la critica jesiana è rivolta agli aspetti intrinsecamente conservatori di un pensiero che ha i tratti dell'ermetismo e che, coltivando l'immagine oscura del passato greco-tedesco, presuppone la distinzione tra la massa inautentica e chi è capace della verità. Il sospetto verso il misticismo riguarda in particolare la concezione heideggeriana del linguaggio poetico: anche in questo caso l'alternativa teorica non poteva che essere la filosofia del linguaggio di Benjamin $^{70}$.

Dall'altro lato vi è la presa di distanza di Jesi dallo strutturalismo, riconducibile al rifiuto dell'“ottimismo" razionalista a cui questo conduce nel dare luogo a un'«ontologizzazione della struttura» e nel riconfigurare il «ruolo di realtà profonda, revocato alla sostanza e alla vita» rimettendolo alla "combinatoria delle forme simboliche» ${ }^{71}$; per Jesi la "generalità di strutture» implica la tentazione di ricadere in forme di ontologia nel momento in cui alcune costanti della vita sociale finiscono per essere considerate costanti dell'essere, dando luogo a una sorta di «conoscenza per reificazione» ${ }^{72}$.

37 La prefazione si chiude con un richiamo alla dimensione politica, non esente da tratti messianici, già emersa in un saggio dello stesso periodo come Conoscibilità della festa ${ }^{73}$ : qui il critico torinese dichiara di avere «precise certezze politiche e morali su ciò che è la dannazione, ma forti dubbi su ciò che sia la salvezza», da non intendere come 
«l'immagine simmetricamente opposta della dannazione». La ripulsa del fascismo e la demistificazione della cultura di destra sono necessarie ma non implicano che si possa vedere in esse l'opposto simmetrico di un progetto di rivoluzione che Jesi, da marxista libertario attento al dibattito militante degli anni Settanta, auspicava. «Il fascismo specularmente capovolto non è il progresso. Ci vuole qualcosa d'altro» - scrive Jesi in un passo che è tra i più oscuri - che sia tale da intervenire «a discriminare con maggior forza, a produrre con maggior violenza la nozione positiva di un abisso» che sia «intervallo tra mito e mitologia, tra il fantasma che "afferra l'uomo" e l'uomo che agisce producendo qualcosa da sé, di sé» ${ }^{74}$.

Jesi sembra qui evocare, con tonalità benjaminiane, una diversa razionalità in grado di porre le basi di una cultura illuminista, progressista e di sinistra adeguata ai tempi ${ }^{75}$. Preparare le condizioni rivoluzionarie significa prima di tutto riconfigurare il rapporto con il mito, riconoscere la paradossale elusività del rapporto tra l'inconoscibile e i suoi effetti, pensare in termini materialistici e antropologici i fondamenti dell'autoaffermazione umana. È riconducibile alla medesima logica un passaggio significativo in cui, negli stessi anni, Jesi parla del «corto circuito mitogenetico» in cui l'essere umano

si sente afferrato perché si afferra. L'uomo non va incontro a una sua presunta fondazione extraumana in forme perenni; va incontro alla sua fondazione in quanto si fonda, contemplando in figure che egli stesso inventa il proprio esser fuso col mondo ${ }^{76}$.

\section{Una scienza come creazione artistica}

La Prefazione (6) ${ }^{77}$, dai forti tratti autobiografici, ha attirato in modo particolare l'attenzione dei commentatori ${ }^{78}$ e pare tracciare una continuità nell'opera dello studioso torinese mostrando il modo in cui riflessione teorica e pratica letteraria si sono intrecciate e contaminate. Come in altri passi Jesi esprime il proprio scetticismo riguardo l'idea di poter formulare qualsivoglia teoria di ordine generale. Dichiara nuovamente di rivolgersi a precisi contesti «nello spazio e nel tempo» ponendosi come obiettivo reale la conoscenza del modo in cui la considerazione dei materiali mitologici si è attuata in diversi momenti storici e «si attua oggi»; il che vuol dire adoperare $\mathrm{i}$ materiali per «conoscerne altri» e per illuminare le connessioni con le «situazioni in cui essi interagirono o interagiscono con determinate modalità di conoscenza» ${ }^{79}$.

Minimizzando la sua produzione teorica Jesi afferma che, qualora abbia dato l'impressione di esprimere giudizi che andassero oltre il dato contestuale e contingente, egli si è in realtà sdoppiato in un sosia che

ha assunto per mimesi le sembianze e il comportamento dello studioso contemporaneo convinto di dire il vero e il giusto, e ha collocato questo sua sosia, loquente con sicurezza di fronte, al suo altro io, disposto ad accettare soltanto temporanee parvenze di probabilità. [...] Alla base della tecnica di conoscenza per composizione sta questo intreccio di due voci, che non può essere dialettico se non nella misura in cui "dialettico" significa puramente e semplicemente "drammatico". L'operazione gnoseologica che si compie in queste pagine è dunque, nelle intenzioni dell'autore - che valgono quel che valgono, ma che è opportuno siano dichiarate -, di natura paradossale, scientifica $e$ artistica. Alla domanda: Non le viene voglia di scrivere un romanzo? l'autore di questo libro può solo rispondere: Non smetto mai di scriverlo ${ }^{80}$.

41 Senza dimenticare che Jesi non ha pubblicato questa pagina, il passo può rivelare un certo dissidio interiore tra la creatività libera del romanziere e il trattenuto rigore dello 
studioso che avvertiva fin dagli esordi: una tensione che risulta una determinante del suo tratto di scrittura in cui si concentrano vasti interessi culturali, spinte politiche e motivi autobiografici. In una lettera del 4 agosto 1970 Jesi scrive a Giulio Schiavoni: «Ho imparato ora anche un'altra tecnica: quella dell'essay più o meno cifrato, che è ben accetta anche alle persone serie non proprio papirologhe. [...] Si accettano stile tono e note, e si adoperano per combattere chi vi attribuisce un valore». Nelle stesse pagine, ancora a proposito del rapporto tra lavoro intellettuale e impegno politico, professa un «marranismo programmatico» capace di «scalzare davvero dalle radici quello che non crediamo giusto» ${ }^{81}$. La critica letteraria praticata negli anni Settanta può essere vista dunque come la forma più adeguata per sciogliere felicemente ogni tensione interna nella direzione dell'elaborazione di uno stile personale quanto riconoscibile. Altrove Jesi ha parlato della necessità di

elaborare il metodo gnoseologico meno coraggioso possibile. Qualsiasi concessione al coraggio dell'“è così" mi sembra una concessione ai meccanismi di alienazione, di oppressione, di potere insomma, che hanno protetto e proteggono la maturazione e l'esplicazione della nostra interiorità e della nostra cultura ${ }^{82}$.

Lo studio della mitologia lo ha portato a postulare la macchina mitologica, pur nella forma di bozza "a matita", e a pensare la pratica intellettuale come un movimento che «mantenga vivo [...] il senso della nostra estraneità» e "permetta di circoscrivere dall'esterno (non certo di penetrare intimamente) il complesso di emozioni, pensieri, esperienze di vita, convenzioni e scelte ${ }^{83}$ connesso al cercare.

L'interpretazione creativa è il modo in cui Jesi ha fatto propria l'ispirazione al tempo stesso materialistica e messianica che caratterizza l'opera di Benjamin. La forma espressiva del saggio esprime nel modo più adeguato un rapporto con il passato colto nell'attualità dell'"ora": Jesi riteneva «inutile, inopportuno e vacuo studiare un testo poetico senza adoperarlo» ${ }^{84}$, intendendolo come uno specchio per conoscere il presente e agire su di esso. Il compito della critica e lo scrivere in generale significano per lui, come si legge proprio in un saggio su Benjamin, «fare della propria pagina lo spazio tristemente privilegiato entro il quale il passato diviene, nella sua cosità, versione interlineare del presente» ${ }^{85}$.

Nell'operazione "scientifica $e$ artistica" condotta da Jesi con i suoi studi si mostra il suo essere un intellettuale militante attivo in molteplici ambiti di riflessione, uno «scrittore di idee e problemi $»^{86}$ capace di interrogarsi sulla capacità della cultura di costruire mondi e sul gioco di caleidoscopica ricomposizione dei materiali che costituisce il come della loro invenzione.

\section{BIBLIOGRAFIA}

AGAMBEN 1999: G. Agamben, «Sull'impossibilità di dire “io”. Paradigmi epistemologici e paradigmi poetici in Furio Jesi», Cultura tedesca 12 (1999), 11-20.

BELPOLITI 2008: M. Belpoliti, «Le rocce fantasma di Furio Jesi», in Id., Diario dell'occhio, Firenze 2008, 104-105. 
BENJAMIN 1962: W. Benjamin, Angelus Novus, trad. it., Torino 1962 (ed. or. 1955 Frankfurt am Main). BIDUSSA 1995: D. Bidussa, «Il vissuto mitologico», in F. Jesi, Germania segreta, Milano 1995, 203-230. CAVALlettr 2001: A. Cavalletti, «La maniera compositiva di Furio Jesi», in Jesi 2001, 359-376. CAVAlLETTI 2002: A. Cavalletti, «Il "romanzo" di Furio Jesi», in F. Jesi, Letteratura e mito, Torino 2002, 245-261.

ECO 1994: U. Eco, La struttura assente, Milano 1994 (prima ed. 1968).

FERRARI 2007: R. Ferrari, Saggio e romanzo in Furio Jesi, Tesi di dottorato in Scienze dell'Antichità e Filologico letterarie dell'Università di Genova, XIX ciclo.

FERRARI 2009: R. Ferrari, «La macchina da scrivere di Furio Jesi», Nuova corrente 143 (2009), 7-34.

FERRARIS 1988: M. Ferraris, «Estetica, ermeneutica, epistemologia», in S. Givone (ed.), Storia dell' estetica, Roma-Bari 1988, 175-221.

HABERMAS 1974: J. Habermas, «Attualità di Walter Benjamin», Comunità 171 (1974), 211-245 (trad. it. di F. Jesi).

JESI 1972: F. Jesi, Thomas Mann, Firenze 1972.

JESI 1973: F. Jesi, Mito, Milano 1973.

JESI 1976: F. Jesi, La festa. Antropologia etnologia folklore, Torino 1976.

JESI 1979: F. Jesi, «Introduzione», in K. Kerényi, Miti e misteri, Torino 1979, 7-25.

JESI 1981: s.v. Walter Benjamin, in Enciclopedia Garzanti di Filosofia, Milano 1981, 83-84.

JESI 1987: F. Jesi, «Il testo come versione interlineare del commento», in Caleidoscopio benjaminiano, in E. Rutigliano e G. Schiavoni (eds.), Roma 1987, 217-220.

JESI 1996: F. Jesi, Lettura del "Bateau Ivre" di Rimbaud, Macerata 1996 (prima ed. 1972); ora in Jesi 2013, 30-58.

JESI 1999: F. Jesi, «Scegliere secondo giustizia (lettere a G. Schiavoni)», Cultura tedesca 12 (1999), 167-181.

JESI 2001: F. Jesi, Materiali mitologici. Mito e antropologia nella cultura mitteleuropea, Torino 2001 (prima ed. 1979).

JESI 2002: F. Jesi, Esoterismo e linguaggio mitologico. Studi su R. M. Rilke, Macerata 2002 (prima ed. 1976).

JESI 2011: F. Jesi, Cultura di destra, Roma 2011 (prima ed. 1979).

JESI 2013: F. Jesi, Il tempo della festa, Roma 2011.

MANERA 2012: E. Manera, Furio Jesi. Mito, violenza, memoria, Roma 2012.

MANERA 2018: E. Manera, «Di ciò che non c'é. “Scienza del mito” e "cultura di destra" in Furio Jesi» , Studi e materiali per la storia delle religioni 84, 1 (2018), 234-253.

MAZZARELLA 1992: E. Mazzarella, «L'istanza cosmologica tra scienza-tecnica e filosofia», Aut aut 250 (1992), 116-123.

TENUTA 2010: C. Tenuta, «“Non smetto mai di scriverlo”: Furio Jesi tra saggistica e narrativa», Intersezioni 3 (2010), 413-438. 


\section{NOTE}

1. JESI 1973, 105.

2. JESI 1996, 28.

3. JESI 1973, 108.

4. Ivi, 109.

5. Ivi, 35-36; 82 ss.

6. JESI 2001,4, n. 6.

7. Cfr. MANERA 2018.

8. JESI $2002,34$.

9. JESI 2011.

10. JESI 1973, 8.

11. JESI 1977, 174-201; JESI 2001, 81-120. La prima pubblicazione è in «Comunità», 169 (1973),

317-347.

12. JESI $2001,94-95$.

13. Ivi, 3-80.

14. JESI 1976.

15. MANERA 2012, 48 ss; 105 ss.;

16. JESI 2001, 102.

17. AGAMBEN 1999, 12.

18. JESI 2001, 116.

19. Ivi, 109.

20. Ivi, 111 .

21. Ivi, 112 .

22. Ivi, 113.

23. Per una più diffusa analisi del concetto di «macchina mitologica» e delle sua diverse formulazione cfr. MANERA 2012, 89 ss.

24. cavalletti 2001, 360: la citazione interna è da una lettera di Jesi a Giulio Schiavoni del 26 giugno 1972.

25. JESI 1973, 8-9; JESI 1996, 36-37; JESI 2001, 206.

26. Cfr. JESI 2001, 174: «La "macchina mitologica" risulta così mitologica perché rientra tra i materiali della mitologia, non perché serva a conoscerli».

27. Ivi, 370, altro frammento inedito contenuto nella cartella La macchina mitologica.

28. Appendice a Jesi 2001, 335-356, a cura di A. Cavalletti. Cfr. la Nota del curatore, 336, e un indice alternativo dell'opera, 372-373.

29. JESI 2001, 337-340. L'ordine di pubblicazione nell'Appendice rispetta la collocazione originaria nella cartella di dattiloscritti.

30. Ivi, 338.

31. Ivi, 339.

32. Ivi, 340 .

33. Ibidem.

34. Cfr. JESI 2011.

35. JESI 2001, 339.

36. FERRARI 2007, 107.

37. BENJAMIN 1962, 83; Jesi usa comunemente la traduzione di Renato Solmi di Jetzeit («attualità»); cfr. Habermas 1974.

38. Jesi $2001,340$.

39. Ibidem.

40. Ivi, 341-342. La numerazione è mia e puramente funzionale. 
41. Ivi, 341.

42. Ivi, 341-342, il termine «è usato nel suo significato tecnico marxista».

43. Ivi, 341 .

44. JESI 1972; JESI 2001, 183-271.

45. Cfr. JESI 2001, 38-50: la stanza del mitologo è hortus conclusus che apre mondi.

46. JESI 2002, 189.

47. JESI $2001,44$.

48. Ivi, 342-345.

49. Ivi, 344 .

50. Ibidem.

51. Ivi, 78-80: la Ferne caratterizza l'opera di Rilke e di Kerényi.

52. Ivi, 344 .

53. Ivi, 345 .

54. Ivi, 346-8.

55. Ivi, 346 .

56. Ivi, p. 347.

57. Ivi, 347. Il riferimento è alla filosofia della storia di Benjamin: cfr. BENJAMIN 1962, 80-84.

58. Ivi, 347; cfr. 353-354.

59. Ivi, 349-350.

60. Ivi, 349 .

61. Ivi, 349-50.

62. Cfr. MANERA 2018.

63. JESI 2001, 350.

64. BIDUSSA 1995, 205.

65. JESI 2001, 351-352

66. Ivi, 351.

67. Ivi, 352 .

68. Ibidem.

69. MAZZARELLA 1992, 116.

70. JESI 2002, 33-34, 167 ss.; JESI 1973, 46-47.

71. FERRARIS 1988, 195.

72. JESI 2001, 98. Cfr. ECO 1994, II-V, X, 295 ss.

73. JESI 1976, 4-29. Ora in JESI 2013, 61-113.

74. JESI 2001, 352.

75. Cfr. JESI 1981, 84: «La redenzione dell'uomo può giungere soltanto da una rottura radicale con il passato improntato dal dominio e da un recupero della tradizione sacra, messianica. Ma in mancanza di elementi di fede come i presupposti della liberazione-redenzione non sono dati, così anche la soggettività liberante attende di essere istituita».

76. JESI 1979, 17.

77. JESI 2001, 353-356,

78. CAVALLETTI 2002; FERRARI 2007, 2009; TENUTA 2010.

79. JESI 2001, 354.

80. Ivi, 355-356.

81. JESI 1999, 177-178.

82. JESI 2002, 43.

83. JESI 1978, 33.

84. JESI 2002, 37.

85. JESI 1987, 219. 


\section{RIASSUNTI}

Il presente articolo è dedicato alla "scienza del mito" in Furio Jesi (1941-1980) e affronta il pensiero epistemologico dell'autore. Il saggio si focalizza sull'originale contributo di Jesi allo studio scientifico del mito e della mitologia mediante il concetto di "macchina mitologica", elaborato nel volume Materiali mitologici (1979) e in particolare in alcuni testi allora non pubblicati e provenienti dall'archivio.

The present paper deals with the "science of myth" in Furio Jesi's (1941-1980) works and intellectual biography. The article describes Jesi's epistemological approach concerning the scientific study of myth and mythology, with a particular focus on the concept of "mythological machine", as it appears in Materiali mitologici (1979) and in some unpublished papers from his archives.

\section{INDICE}

Keywords : myth, critics of ideology, history of historiography, epistemology of humanities, literary criticism

Parole chiave : mito, critica dell'ideologia, storia della storiografia, epistemologia delle scienze umane, critica letteraria

\section{AUTORE}

\section{ENRICO MANERA}

Istituto piemontese per la storia della Resistenza e della società contemporanea G. Agosti, Via del Carmine, 13, 10122 Torino, Italia enrico.manera(at)istoreto.it 